\title{
Propofol alleviates acute lung injury following orthotopic autologous liver transplantation in rats via inhibition of the NADPH oxidase pathway
}

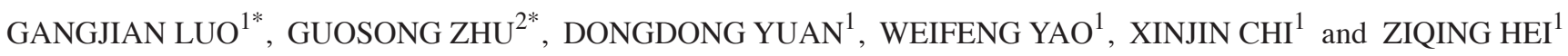 \\ ${ }^{1}$ Department of Anesthesiology, The Third Affiliated Hospital of Sun Yat-sen University, Guangzhou, \\ Guangdong 510630; ${ }^{2}$ Department of Anesthesiology, Henan Provincial People's Hospital, \\ Zhengzhou, Henan 450003, P.R. China
}

Received December 28, 2013; Accepted October 31, 2014

DOI: $10.3892 / \mathrm{mmr} .2014 .2924$

\begin{abstract}
Acute lung injury (ALI) induced by liver transplantation is detrimental to patient survival, and therapeutic strategies remain limited. Thus, the protective effects of propofol, a commonly used anesthetic with antioxidative and anti-inflammatory properties, were investigated in the present study on ALI induced by orthotopic autologous liver transplantation (OALT). The protective mechanism of propofol was determined to be associated with the inhibition of NADPH oxidase, by comparing its effects with the positive controls apocynin (AP; an NADPH oxidase inhibitor) and N-acegysteine (NAC; a scavenger of reactive oxygen species). The results demonstrated that two proteins (p47phox and gp91phox) of the NADPH oxidase system presented increased expression in rats with ALI induced by OALT, thus leading to increased activation of the oxidative stress and inflammatory reactions. Preconditioning with NAC or AP eliminated this increase, suggesting that antioxidative treatment, particularly with inhibitors of NADPH oxidase, is a promising protective strategy against ALI induced by OALT. Propofol preconditioning at a high $(100 \mathrm{mg} / \mathrm{kg})$ or low $(50 \mathrm{mg} / \mathrm{kg})$ dose promoted similar protective effects, with the high-dose propofol producing a more marked effect than the low dose. The results suggested that propofol may protect against ALI induced by OALT, the mechanism of which may involve a reduced oxidative stress and inflammatory reaction mediated by NADPH oxidase inhibition.
\end{abstract}

Correspondence to: Professor Ziqing Hei or Dr Dongdong Yaun, Department of Anesthesiology, The Third Affiliated Hospital of Sun Yat-sen University, 600 Tianhe Road, Guangzhou, Guangdong 510630, P.R. China

E-mail: heiziqing@sina.com

E-mail: yuandongdong123@126.com

*Contributed equally

Key words: propofol, autologous orthotropic liver transplantation, acute lung injury, NAPDH

\section{Introduction}

Liver transplantation is considered to be the most effective treatment for end-stage liver disease. However, a variety of postoperative complications severely affect patient survival, one of which is acute lung injury (ALI). It has been indicated that the incidence of ALI in liver transplantation patients is 34.2 77.8\% (1). Among patients with ALI, those who develop acute respiratory distress syndrome (ARDS) have a mortality rate of $76.5 \%$. Thus, the treatment of complications in the lungs is crucial to the recovery of patients undergoing liver transplantation. Strategies used to protect the lungs in this operation, however, remain limited, thus it is important to determine novel protective methods.

During liver transplantation, the inferior vena cava (IVC) and portal vein (PV) are interrupted, which causes hypotension and intestinal congestion, resulting in ischemia-reperfusion injury. Reactive oxygen species (ROS), endotoxins and cytokines enter into the circulation and damage remote organs and systems, of which the lungs are considered to be the most vulnerable (2). Therefore, therapeutic strategies alleviating ALI induced by liver transplantation are required to improve patient prognosis.

Oxidative stress and inflammation are considered as the main causes of ALI (3), and previous studies have provided evidence that oxidative stress or inflammatory damage serve an important function in this pathological process $(4,5)$. Thus, antioxidative therapies for lung protection require investigation, particularly in liver transplantation (6). NADPH oxidase is one of the key enzymes in ROS production; its inhibition may be effective in reducing the levels of ROS, thus further reducing oxidative stress and inflammation (7). Therapeutic strategies targeting NADPH oxidase have not been widely studied, and few have been utilized clinically.

Propofol, an intravenous anesthetic with a hydroxyl group attached to its benzene ring (phenol) (8), is widely used clinically. The antioxidant and anti-inflammatory properties of propofol, which are considered to be due to its phenolic hydroxyl group, have become a focus of recent research. However, whether propofol can effectively attenuate ALI induced by liver transplantation remains unclear. Thus, in 
the current study, a rat OALT model was used to explore the protective effects of propofol on ALI induced by liver transplantation compared with positive controls, including: NAC [a non-specific antioxidant (14)] and AP [a specific NADPH oxidase inhibitor (15)]. The mechanisms of the protective effect of propofol were analyzed, and suggested to be associated with the reduction in oxidative stress and inflammatory reaction mediated by the inhibition of NADPH oxidase.

\section{Materials and methods}

Animals. The National Institutes of Health criteria for the care and use of laboratory animals in research was followed. The study was approved by the Laboratory Animal Care Committee of Sun Yat-Sen University (Guangzhou, China). Adult specific pathogen-free male Sprague-Dawley rats (body weight, 180-220 g) were purchased from the Laboratory Animal Center of Sun Yat-Sen University.

Rat OALT model establishment. Rats were fasted for $8 \mathrm{~h}$ with free access to drinking water prior to surgery, and were subsequently injected with $1 \mathrm{mg} / \mathrm{kg}$ atropine (Nanjing Keygen Biotech Co., Ltd, Nanjing, China) 15 min prior to surgery in order to prevent secretion, which may lead to asphyxia in rats. An open face guard was used to administer $2 \%$ ether anesthetic via inhalation (Nanjing Keygen Biotech Co., Ltd). The constructed OALT model was similar to that described previously $(9,10)$. Subsequent to entering the abdominal cavity, the falciform ligament of the liver was resected and ligated, and the left vena phrenica along the esophagus was severed. The liver was exposed until the superior vena cava (SVC) was completely freed. Silk thread was use to raise the freed SVC slightly, in order to be easily blocked using vascular clamps at a later stage. The liver was then placed back in its original position and the IVC was dissected until the upper region of the left renal vein was completely separated. The first hepatic portal was dissected and the PV was separated from the convergence of the inferior mesenteric and splenic veins. The hepatic artery and biliary tract were dissected and separated together due to their anatomical relationship. The hepatic portal veins were ligated. Vascular clamps were used on the hepatic artery, SVC, IVC and at the convergence of the inferior mesenteric and splenic veins. The PV was punctured with a 24-gauge needle and fixed in place with a vascular clamp in preparation for reperfusion. Ringer's lactate solution (Jetway Biotech Co., Ltd, Guangzhou, China), precooled to $4^{\circ} \mathrm{C}$, was injected during reperfusion at $2.5 \mathrm{ml} / \mathrm{min}$ and a 1-mm incision was made on the wall of the IVC as an outflow tract. The needle was then extracted and the openings of the PV and the IVC were closed using 8-0 sutures and the PV, SVC, IVC and hepatic artery were unclamped. The anhepatic phase lasted for $20 \pm 1 \mathrm{~min}$.

Animal groups. The experimental animals were randomly divided into seven groups using a random number table, which considered the weights of the animals, as follows ( $n=8$ in each group): The sham (S), model with saline (M), fat milk (FM), low propofol dose (LP), high propofol dose (HP), NAC (NC) and apocynin (AP) groups. Groups S and M were injected with $2 \mathrm{ml} /$ day physiological saline (Jetway Biotech Co., Ltd) for three consecutive days. Groups FM,
Table I. Rat body weight and anhepatic phase duration time.

\begin{tabular}{llc}
\hline Group & Weight $(\mathrm{g})$ & Anhepatic phase (min) \\
\hline S & $198.3 \pm 10.3$ & - \\
M & $202.6 \pm 9.7$ & $19.7 \pm 0.5$ \\
FM & $213.4 \pm 12.3$ & $20.0 \pm 0.9$ \\
LP & $195.1 \pm 15.4$ & $19.9 \pm 1.1$ \\
HP & $208.3 \pm 18.0$ & $19.3 \pm 0.6$ \\
NC & $199.2 \pm 13.3$ & $20.5 \pm 0.3$ \\
AP & $215.8 \pm 19.2$ & $19.4 \pm 0.5$ \\
\hline
\end{tabular}

LP, HP, NC and AP were injected with fat milk [2 ml/day; intraperitoneal (i.p.) injection; Mitsubishi Pharma Co., Ltd, Guangzhou, China], propofol (50 mg/kg; i.p.; $1 \%$ Diprivan; CG411; AstraZeneca, Caponago, Italy), at previously determinend doses (11); propofol (100 mg/kg; i.p.), NAC (150 mg/kg; i.p; Sigma-Aldrich, St. Louis, MO, USA) (12); or apocynin (5 mg/kg; i.p.; Sigma-Aldrich) (13), respectively, for three consecutive days. All drugs were diluted in $2 \mathrm{ml}$ physiological saline. On the fourth day, the rats in group $\mathrm{S}$ were subject to celiotomy and vascular separation under anesthesia. The rats in the other groups underwent the OALT operation. Lung tissues were collected $8 \mathrm{~h}$ subsequent to liver transplantation (10).

The rat body weights and the anhepatic phase duration times in the seven groups are displayed in Table I, with no significant differences observed between groups $(\mathrm{P}>0.05)$.

Disposal of specimens. The animals were anesthetized via intraperitoneal injection of $10 \%$ chloral hydrate $(3.5 \mathrm{ml} / \mathrm{kg}$; Jetway Biotech Co., Ltd) 8 h subsequent to OALT; subsequently, $2 \mathrm{ml}$ air was injected into the tail vein in order to sacrifice the rats. The thorax was opened and all lung tissues were removed. The middle lobe of the right lung was weighed on an electronic scale and the inferior lobe was fixed in $10 \%$ buffered formalin (Nanjing Keygen Biotech Co., Ltd) and embedded in paraffin (Nanjing Keygen Biotech Co., Ltd) for histological evaluation. The remaining lung tissue was promptly transferred into liquid nitrogen (Nanjing Keygen Biotech Co., Ltd) for storage until it was required for $\mathrm{p} 47 \mathrm{phox}$ and gp91phox expression assays and the measurement of hydrogen peroxide $\left(\mathrm{H}_{2} \mathrm{O}_{2}\right)$, malondialdehyde (MDA) and superoxide dismutase (SOD) activity.

Lung histology. The lung tissues were sectioned ( 4 mm) and stained with hematoxylin and eosin (H\&E; Nanjing Keygen Biotech Co., Ltd). The sample groups were analyzed blindly and the pathology was scored as described by Franco-Gou et al (16). The graded edema of the alveolar mesenchyme, intra-alveolar cell infiltration and alveolar hemorrhage were also scored.

Lung water content. The wet weight of the superior lobe of the right lung was measured, and then the samples were placed in an oven for $24 \mathrm{~h}$ at $80^{\circ} \mathrm{C}$ in order for them to dry out; once water was evaporated the tissue reached a constant weight. The water content of the lung was calculated as follows: 
A

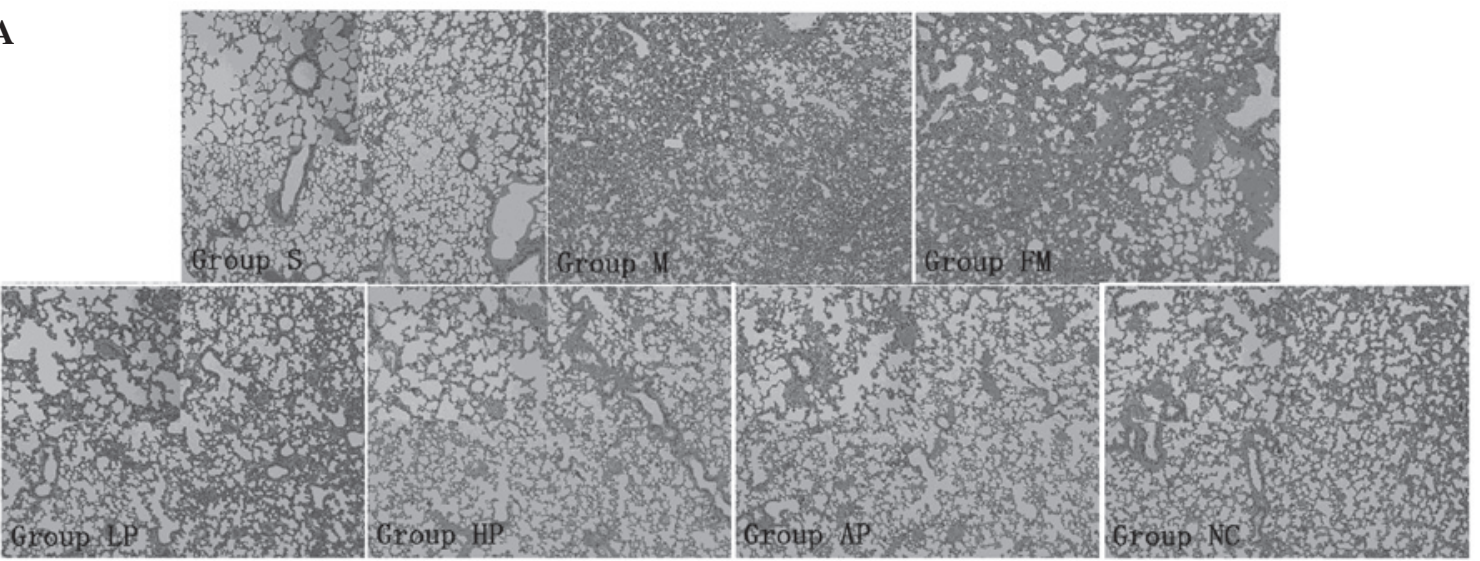

B

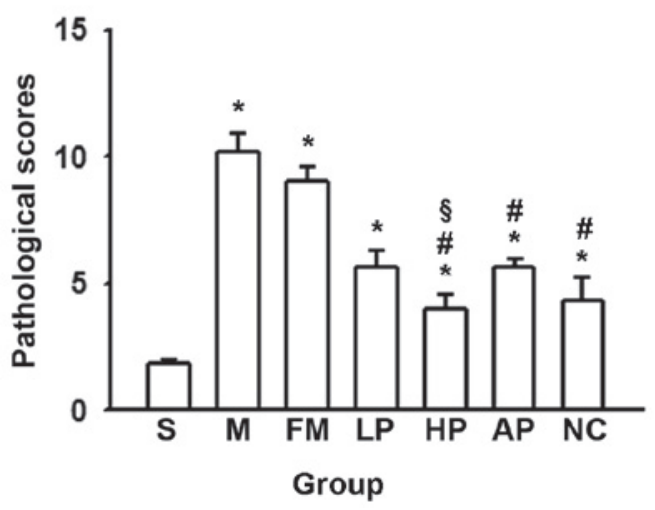

C

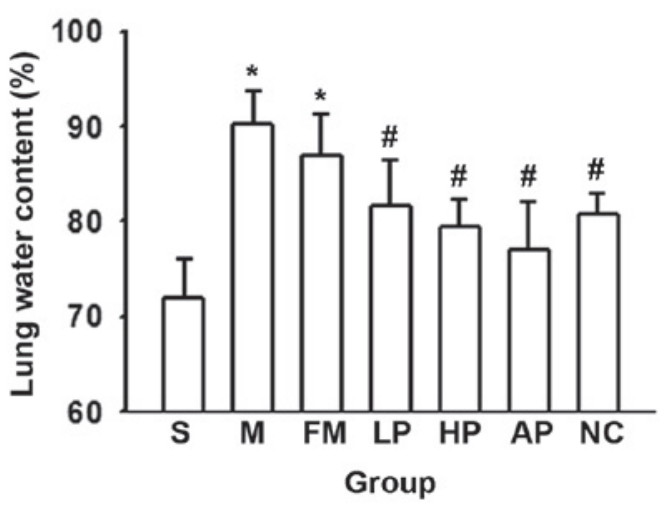

Figure 1. Pathological alterations in lung tissue following OALT. (A) Histological pathological changes in lung tissue. Magnification, x100. (B) Lung pathological scores. (C) Alterations in water content of the lung tissues following OALT. Data are presented as the mean \pm standard deviation ( $\mathrm{n}=8$ in each group). ${ }^{*} \mathrm{P}<0.05$ vs. group $\mathrm{S},{ }^{~} \mathrm{P}<0.05$ vs. group $\mathrm{M},{ }^{\S} \mathrm{P}<0.05$ vs. group LP. S, sham; M, saline control; FM, fat milk control; LP, low-dose propofol; HP, high-dose propofol; NC, N-acegysteine positive control; AP, apocynin positive control; OALT, orthotopic autologous liver transplantation.

Water content $=($ lung wet weight - lung dry weight $) /$ lung wet weight x 100 (11).

Western blot analysis. Lung tissues were finely homogenized, suspended in ice-cold lysis buffer $(1.5 \mathrm{ml} / \mathrm{g}$ tissue; Nanjing Keygen Biotech Co., Ltd) and then centrifuged $(12,000 \mathrm{x} \mathrm{g}$ for $10 \mathrm{~min}$ at $4{ }^{\circ} \mathrm{C}$ ). The supernatants were collected for analysis. Following measurement of the protein concentration of each sample, $50 \mu \mathrm{g}$ of the sample was solubilized in sodium dodecyl sulfate (SDS) loading buffer (Bio-Rad Laboratories, Inc., Hercules, CA, USA) by boiling. The samples were loaded onto a $10 \%$ polyacrylamide gel (Invitrogen Life Technologies, Carlsbad, CA, USA) and SDS-PAGE (Bio-rad Laboratories, Inc.) was conducted. They were then transferred to a polyvinylidene difluoride (PVDF; Bio-rad Laboratories, Inc.) membrane. The PVDF membrane was subsequently incubated with monoclonal mouse anti-human p47-phox (sc-17845) and gp91-phox (sc-74514) antibodies (1:500; Santa Cruz Biotechnology, Inc., Dallas, TX, USA) as well as monoclonal mouse Immunoglobulin G1 anti- $\beta$-actin (1:8,000; A5441; Sigma-Aldrich), followed by the corresponding horseradish peroxidase-conjugated secondary antibodies (1:2,00; Santa Cruz Biotechnology, Inc.). Protein-antibody complexes were detected with an enhanced chemiluminescence system (KGP1125; Nanjing Keygen Biotech). Protein band sizes were estimated using AlphaView 2.2.14407 software (ProteinSimple, Santa Clara,
CA, USA). The density measurement was correlated to the protein expression and normalized to $\beta$-actin.

Detection of $\mathrm{H}_{2} \mathrm{O}_{2}, M D A, S O D$, tumor necrosis factor- $\alpha$ (TNF- $\alpha$ ) and interleukin-6 (IL-6). $\mathrm{H}_{2} \mathrm{O}_{2}$, MDA and SOD levels were measured using the corresponding kits $\left(\mathrm{H}_{2} \mathrm{O}_{2}\right.$ kit, MDA kit and SOD assay kit; all purchased from Nanjing Keygen Biotech. Co., Ltd) according to the manufacturer's instruction. The concentrations of TNF- $\alpha$ and IL- 6 were measured using respective ELISA kits (Nanjing Keygen Biotech. Co., Ltd.).

Statistical analysis. All data are presented as the mean \pm standard error and analyzed using SPSS software, version 12.0 (SPSS, Inc., Chicago, IL, USA). The differences between groups were analyzed using one-way ANOVA. $\mathrm{P}<0.05$ was considered to indicate a statistically significant difference.

\section{Results}

Propofol protects against OALT-induced changes in lung morphology. Alterations in lung morphology following OALT were estimated using H\&E staining. Greater lung damage was observed in group $M$ than group $S$ (blood vessel liberation only). Clear inflammatory cell infiltration and alveolar exudates were observed and the pulmonary interstitium exhibited hyperemia and severe hemorrhage 
A
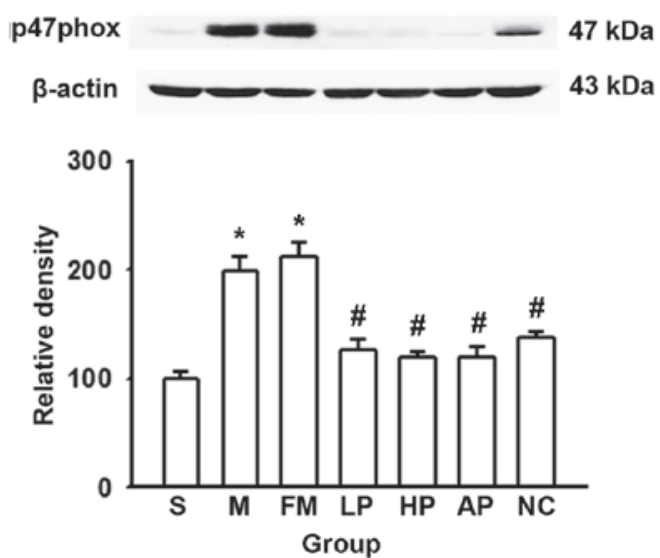

B
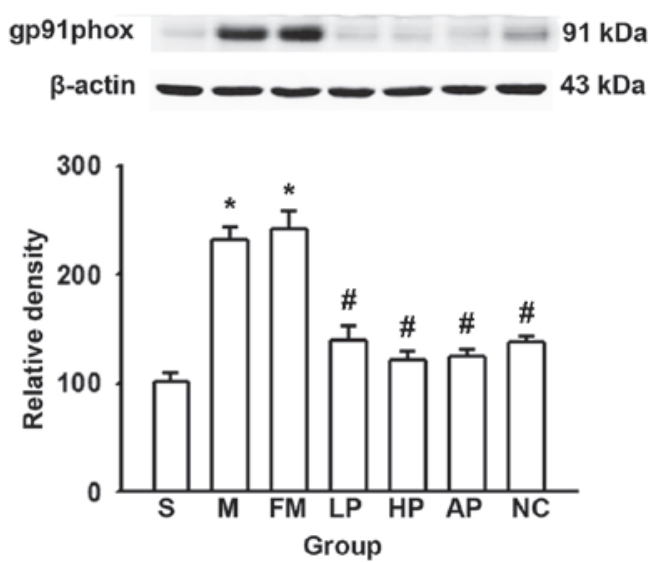

Figure 2. Expression levels of the NADPH oxidase subunits p47phox and gp91phox in lung tissues following OALT. (A) Relative density of p47phox to $\beta$-actin expression. (B) Relative density of gp91phox to $\beta$-actin expression. Data are presented as the mean \pm standard deviation ( $\mathrm{n}=8$ in each group). " $\mathrm{P}<0.05 \mathrm{vs}$. group $\mathrm{S},{ }^{\#} \mathrm{P}<0.05$ vs. group $\mathrm{M},{ }^{\circledR} \mathrm{P}<0.05$ vs. group AP. S, sham; M, saline control; FM, fat milk control; LP, low-dose propofol; HP, high-dose propofol; NC, $\mathrm{N}$-acegysteine positive control; AP, apocynin positive control; OALT, orthotopic autologous liver transplantation.
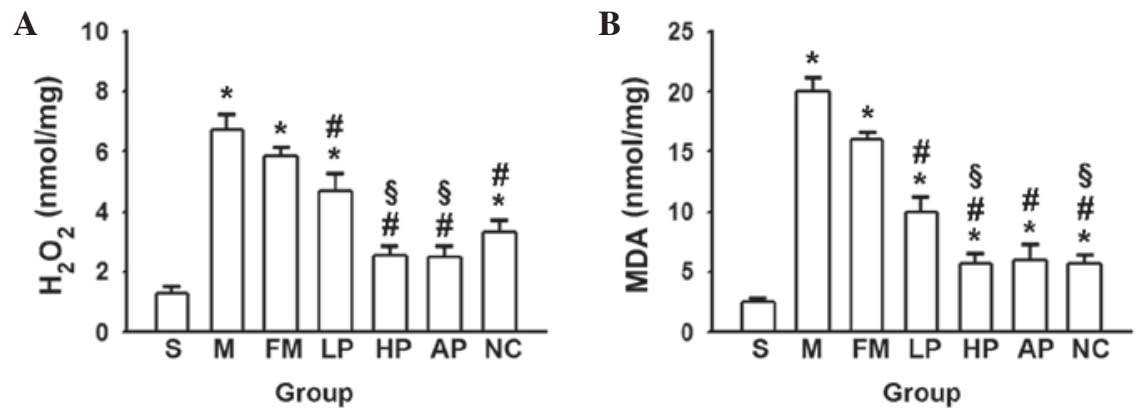
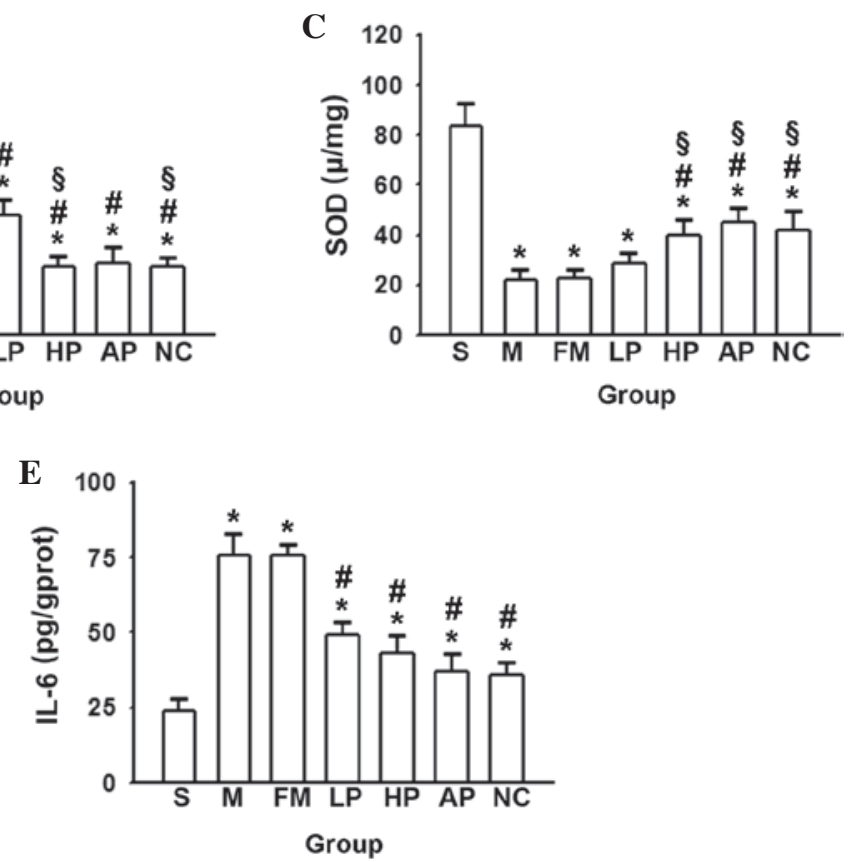

Figure 3. Concentration of (A) $\mathrm{H}_{2} \mathrm{O}_{2}$, (B) MDA, (C) SOD, (D) TNF- $\alpha$ and (E) IL-6 following OALT. Data are presented as the mean \pm standard deviation ( $\mathrm{n}=8$ in each group). ${ }^{*} \mathrm{P}<0.05$ vs. group $\mathrm{S},{ }^{\#} \mathrm{P}<0.05$ vs. group $\mathrm{M},{ }^{\S} \mathrm{P}<0.05$ vs. group $\mathrm{LP} \mathrm{H}_{2} \mathrm{O}_{2}$, hydrogen peroxide; MDA, malondialdehyde; SOD, superoxide dismutase; TNF- $\alpha$, tumor necrosis factor- $\alpha$; IL-6, interleukin 6; S, sham; M, saline control; FM, fat milk control; LP, low-dose propofol; HP, high-dose propofol; $\mathrm{NC}, \mathrm{N}$-acegysteine positive control; AP, apocynin positive control; OALT, orthotopic autologous liver transplantation.

(Fig. 1A and B) (16). Propofol, at low (50 mg/kg) and high (100 $\mathrm{mg} / \mathrm{kg})$ doses, significantly protected against lung damage, producing similar results as the two established antioxidants, NAC and AP. In addition, the high-dose propofol was more efficacious than the low-dose, and as a solvent control of propofol, fat milk did not exhibit any protective effects on lung tissue (group FM).

Propofol reduces lung water content. The water content of the middle lobe of the right lung was measured in order to estimate the extent of pulmonary edema following OALT, as this measure is indicative of pathological damage. Propofol reduced the water content of lungs significantly, producing similar results to NAC and AP (Fig. 1C). The results suggested that ALI induced by liver transplantation was severe, but propofol was able to reverse this damage and attenuate pulmonary edema.

NADPH oxidase protein expression in lung tissue. NADPH oxidase, a key multiprotein system involved in the generation of ROS, consists of cytochrome b558 (gp91phox and p22phox) on the membrane and cytosolic soluble proteins (p67phox, p47phox, p40phox and Rac1/Rac2). The current study focused on the alterations in p47phox and gp91phox levels in the lungs subsequent to OALT, as these proteins are fundamental for the activity of the NADPH oxidase system. AP (an inhibitor of 
NAPDH oxidase) and NAC (a ROS scavenger) were used as positive controls. As demonstrated in Fig. 2, propofol, NAC and AP pretreatment significantly reduced the expression of p47phox and gp91phox induced by OALT, compared with the saline control group, with levels similar to those of the sham group. The results suggested that NADPH oxidase activity is enhanced in lung tissues following liver transplantation. Thus, as propofol produces similar antioxidant effects to NAC and $\mathrm{AP}$, this is thought to be mediated by the inhibition of NADPH oxidase activity.

Alterations in levels of oxidative stress and inflammation in lung tissue following liver transplantation. NADPH oxidase produces large quantities of ROS, which are involved in the processes of oxidative stress, inflammation, cell signal transduction, cell proliferation and apoptosis $(17,18)$. Previous studies have implicated oxidative stress and the inflammatory reaction to serve important functions in the development of ALI $(19,20)$. Thus, alterations to oxidative and inflammatory damage in ALI induced by liver transplantation were focused upon in the present study. The concentrations of $\mathrm{H}_{2} \mathrm{O}_{2}$, MDA and SOD in the lungs subsequent to OALT were determined, which reflects the balance of oxidative stress $(21,22)$. Results indicated a significant increase in the levels of $\mathrm{H}_{2} \mathrm{O}_{2}$ and MDA subsequent to OALT, and propofol, NAC and AP pretreatment reduced this increase. The high-dose propofol produced a significantly reduced level of $\mathrm{H}_{2} \mathrm{O}_{2}$ and MDA compared with the low dose (Fig. 3A and B). However, the opposite effect was observed in the levels of SOD, with the lowest levels observed in the saline and fat milk groups, and an increase following propofol, AP and NAC pretreatment (Fig. 3C). The results suggested that propofol effectively reduced ROS production and alleviated oxidative damage to protect against the lung damage induced by OALT, in a similar manner to the positive controls NAC and AP.

To analyze another aspect, the levels of two cytokines (TNF- $\alpha$ and IL-6) that are important in ALI were measured. Fig. 3D and E illustrates that the levels of TNF- $\alpha$ and IL-6 were reduced by propofol pretreatment prior to OALT compared with levels following saline treatment, similar to the levels observed following NAC and AP pretreatment. This suggests that propofol attenuated OALT-induced inflammatory factors in order to protect the lung tissue against inflammatory damage.

\section{Discussion}

ALI is a major complication of OALT that significantly affects prognosis, resulting in an increased mortality rate (23). Thus, the influence of liver transplantation on remote lung damage was investigated in the present study, with an aim to develop a novel strategy to protect the lungs during this procedure. In the current study, a rat OALT model was used to observe alterations in pathological lung injuries. The rat model closely mimicked the aspects of the liver transplantation procedure, including blockade of the SVC, IVC and PV, cold liver protection, fluid perfusion, liver ischemia-reperfusion injury and passive congestion of the intestine. All of the above contributed to the investigation of the effects of OALT procedures on lung damage, but did not take into account the complex situation of liver rejection. The data demonstrated that ALI induced by liver transplantation was serious, and the ROS induced by NADPH oxidase participated in this process through activating secundum oxidative stress and inflammatory reaction. AP and NAC (inhibitors of NADPH oxidase) preconditioning diminished this damage effectively, and the observation that propofol produced similar antioxidative effects to these suggests that the protective mechanism of propofol involves NADPH oxidase inhibition.

Several studies have illustrated that oxidative stress and the inflammatory reaction are key in the pathogenesis of ALI (24,25). During liver transplantation, the IVC and PV require interruption, which results in hypotension and intestinal congestion, leading to ischemia-reperfusion injury, in which ROS, endotoxins and cytokines enter into the blood circulation and damage remote organs and systems. ROS directly damage lung parenchymal cells via lipid peroxidation, in addition to the basement membrane of capillaries and pulmonary interstitial cells, thereby resulting in severe pulmonary edema (26). Previous studies have indicated that a reduction in ROS production may alleviate liver or lung ischemia-reperfusion injury (27), as a sudden influx of ROS can overwhelm innate protective measures and lead to organ injury (28). ROS are formed through various key enzymes, including xanthine oxidase, NADPH oxidase and nitric oxide synthase (29-31), of which NADPH oxidase-dependent ROS formation is considered to be the most important. Thus, in the current study, the main focus was upon this pathway, to explore the effects of liver transplantation on remote lung damage. NADPH oxidase is present in neutrophils, macrophages and on the membranes of endothelial cells, particularly in pulmonary vasculature where it is prevalent $(31,32)$. Previous studies have demonstrated that the increase in NADPH oxidase is the main mechanism of ischemia-reperfusion injury, and its inhibition with diphenyliodonium (31) and apocynin (15) for example, have markedly reduced ROS formation and tissue damage in previous in vivo studies. Apocynin is a direct inhibitor of NADPH oxidase, and NAC may act as a ROS scavenger by promoting the synthesis of glutathione (33). The observation that two NADPH oxidase inhibitors with different mechanisms yielded similar results suggested that ROS production mediated by NAPDH oxidase serves an important role in ALI induced by OALT. Thus, antioxidant preconditioning reduced the resulting lung damage.

Propofol contains a phenol hydroxyl group that confers antioxidant activity. Takao et al (34) suggested that a high dose of propofol mitigates the physiological, biochemical and histological deterioration of ALI during endotoxemia. Propofol has also been reported to exert significant protective activity against ischemia-reperfusion-induced cardiac injury, partly through the reduction in $\mathrm{ROS}$ and $\mathrm{H}_{2} \mathrm{O}_{2}$ generation $(35,36)$. In the current study, propofol was demonstrated to produce a similar effect to that of NAC and AP on lung tissue; reducing expression of NADPH oxidase (Fig. 2) and levels of $\mathrm{H}_{2} \mathrm{O}_{2}$ and MDA, but increasing the level of SOD (Fig. 3). Propofol was also observed to act as an antioxidant, inhibiting NAPDH oxidase to protect lung tissue from oxidative stress. The results of the current study indicate that propofol, as an antioxidant, can be applied and used clinically.

Excessive inflammation is considered to be one of the underlying mechanisms of the pathogenesis of ALI/ARDS, in which TNF- $\alpha$ and IL-6 are the major cytokines involved (37). Oxidative stress and the inflammatory reaction interact in a 
complex way to act in the pathogenesis of lung damage. Studies have demonstrated that ROS are fundamental to oxidative stress and the inflammatory reaction $(38,39)$. The current study demonstrated that NAC and AP application inhibited NAPDH oxidase in the earlier phase and reduced or eliminated ROS production. This was beneficial for reducing oxidative stress and the inflammatory reaction in the lungs, mediated by OALT. Propofol preconditioning was observed to produce similar effects to NAC or AP, thus propofol was hypothesized to also reduce the inflammatory reaction initiated by ROS through the inhibition of NAPDH oxidase (Fig. 3D and E). An additional study demonstrated that propofol also possessed anti-inflammatory properties, however, it was unknown whether it was able to effect oxidative stress through its anti-inflammatory action (40). Further investigations to further elucidate this are required.

In conclusion, the current study demonstrated that propofol, a common clinically used anesthetic, protects against lung damage via the inhibition of oxidative stress and the inflammatory reaction, particularly via NADPH oxidase inhibition. This may be used as a novel strategy for organ protection during liver transplantation. Further investigation into the optimal protective dose and administration time of propofol should be conducted in future studies.

\section{Acknowledgements}

The current study was supported by the National Natural Science Foundation of China (grant nos. 81401628 and 30972858); the Natural Science Foundation of Guangdong Province, China (grant no. S2012010008930), and the Medical Research Foundation of Guandong Province (grant no. B2014141).

\section{References}

1. Hong SK, Hwang S, Lee SG, et al: Pulmonary complications following adult liver transplantation. Transplantat Proc 38: 2979-2981, 2006.

2. Hirsch J, Niemann CU, Hansen KC, et al: Alterations in the proteome of pulmonary alveolar type II cells in the rat after hepatic ischemia-reperfusion. Crit Care Med 36: 1846-1854, 2008

3. Bellingan GJ: The pulmonary physician in critical care * 6 : The pathogenesis of ALI/ARDS. Thorax 57: 540-546, 2002.

4. Jiang A, Liu C, Song Y, et al: NF- $\kappa$ B induced the donor liver cold preservation related acute lung injury in rat liver transplantation model. PloS One 6: e24960, 2011.

5. Hei Z, Chi X, Cheng N, Luo G and Li S: Upregulation of TLR2/4 expression in mononuclear cells in postoperative systemic inflammatory response syndrome after liver transplantation. Mediators Inflamm 2010: 519589, 2010.

6. Altintas ND, Atilla P, Iskit AB and Topeli A: Long-term simvastatin attenuates lung injury and oxidative stress in murine acute lung injury models induced by oleic Acid and endotoxin. Respir Care 56: 1156-1163, 2011

7. Ramonaite R, Skieceviciene J, Kiudelis G, et al: Influence of NADPH oxidase on inflammatory response in primary intestinal epithelial cells in patients with ulcerative colitis. BMC Gastroenterol 13: 159, 2013.

8. Murphy PG, Myers DS, Davies MJ, Webster NR and Jones JG: The antioxidant potential of propofol (2,6-diisopropylphenol). Br J Anaesth 68: 613-618, 1992.

9. Jin C, Zhang PJ, Wu XM, et al: Impact of hypoxic preconditioning on apoptosis and its possible mechanism in orthotopic liver autotransplantation in rats. Hepatobiliary Pancreat Dis Int 8: 40-45, 2009.

10. Chi X, Zhang A, Luo G, et al: Knockdown of myeloid differentiation protein-2 reduces acute lung injury following orthotopic autologous liver transplantation in a rat model. Pulm Pharmaco Ther 26: 380-387, 2013.
11. Liu KX, Chen SQ, Huang WQ, Li YS, Irwin MG and Xia Z: Propofol pretreatment reduces ceramide production and attenuates intestinal mucosal apoptosis induced by intestinal ischemia/reperfusion in rats. Anesth Analg 107: 1884-1891, 2008.

12. Jin X, Wang L, Wu HS, et al: $\mathrm{N}$-acetylcysteine inhibits activation of toll-like receptor 2 and 4 gene expression in the liver and lung after partial hepatic ischemia-reperfusion injury in mice. Hepatobiliary Pancreat Dis Int 6: 284-289, 2007.

13. Sonta $\mathrm{T}$, Inoguchi $\mathrm{T}$, Tsubouchi $\mathrm{H}$, et al: Evidence for contribution of vascular $\mathrm{NAD}(\mathrm{P}) \mathrm{H}$ oxidase to increased oxidative stress in animal models of diabetes and obesity. Free Radic Biol Med 37: 115-123, 2004.

14. Sadowska AM: N-Acetylcysteine mucolysis in the management of chronic obstructive pulmonary disease. Ther Adv Respir Dis 6: 127-135, 2012.

15. Dodd-o JM, Welsh LE, Salazar JD, et al: Effect of NADPH oxidase inhibition on cardiopulmonary bypass-induced lung injury. Am J Physiol Heart Circ Physiol 287: H927-H936, 2004.

16. Franco-Gou R, Roselló-Catafau J and Peralta C: Protection against lung damage in reduced-size liver transplantation. Crit Care Med 34: 1506-1513, 2006.

17. Fan J, Frey RS and Malik AB: TLR4 signaling induces TLR2 expression in endothelial cells via neutrophil NADPH oxidase. J Clin Invest 112: 1234-1243, 2003.

18. Poinas A, Gaillard J, Vignais P and Doussiere J: Exploration of the diaphorase activity of neutrophil NADPH oxidase. Eur J Biochem 269: 1243-1252, 2002.

19. Auten RL, Richardson RM, White JR, Mason SN, Vozzelli MA and Whorton MH: Nonpeptide CXCR2 antagonist prevents neutrophil accumulation in hyperoxia-exposed newborn rats. J Pharmacol Exp Ther 299: 90-95, 2001.

20. Auten RL, Whorton MH and Nicholas Mason S: Blocking neutrophil influx reduces DNA damage in hyperoxia-exposed newborn rat lung. Am J Respir Cell Mol Biol 26: 391-397, 2002.

21. Hõrak P, Sild E, Soomets U, Sepp T and Kilk K: Oxidative stress and information content of black and yellow plumage coloration: an experiment with greenfinches. J Exp Biol 213: 2225-2233, 2010.

22. Manni ML,Epperly MW, Han W, et al: Leukocyte-derived extracellular superoxide dismutase does not contribute to airspace EC-SOD after interstitial pulmonary injury. Am J Physiol Lung Cell Mol Physiol 302: L160-L166, 2012.

23. Keegan MT and Pickering BW: Critical care issues following orthotopic liver transplantation. Minerva Gastroenterol Dietol 56: 305-330, 2010.

24. Yamaoka S, Kim HS, Ogihara T, et al: Severe Vitamin E deficiency exacerbates acute hyperoxic lung injury associated with increased oxidative stress and inflammation. Free Radic Res 42: 602-612, 2008

25. Weng TI, Wu HY, Kuo CW and Liu SH: Honokiol rescues sepsis-associated acute lung injury and lethality via the inhibition of oxidative stress and inflammation. Intensive Care Med 37: 533-541, 2011.

26. Compton CN, Franko AP, Murray MT, Diebel LN and Dulchavsky SA: Signaling of apoptotic lung injury by lipid hydroperoxides. J Trauma 44: 783-788, 1998.

27. Kupatt C, Habazettl H, Goedecke A, et al: Tumor necrosis factor-alpha contributes to ischemia- and reperfusion-induced endothelial activation in isolated hearts. Circ Res 84: 392-400, 1999.

28. Kennedy TP, Rao NV, Hopkins C, Pennington L, Tolley E and Hoidal JR: Role of reactive oxygen species in reperfusion injury of the rabbit lung. J Clin Invest 83: 1326-1335, 1989.

29. den Hengst WA, Gielis JF, Lin JY, Van Schil PE, De Windt LJ and Moens AL: Lung ischemia-reperfusion injury: a molecular and clinical view on a complex pathophysiological process. Am J Physiol Heart Circ Physiol 299: H1283-H1299, 2010.

30. Adkins WK and Taylor AE: Role of xanthine oxidase and neutrophils in ischemia-reperfusion injury in rabbit lung. J Appl Physiol (1985) 69: 2012-2018, 1990.

31. Al-Mehdi AB, Zhao G, Dodia C, et al: Endothelial NADPH oxidase as the source of oxidants in lungs exposed to ischemia or high $\mathrm{K}^{+}$. Circ Res 83: 730-737, 1998.

32. Zulueta JJ, Yu FS, Hertig IA, Thannickal VJ and Hassoun PM: Release of hydrogen peroxide in response to hypoxia-reoxygenation: role of an $\mathrm{NAD}(\mathrm{P}) \mathrm{H}$ oxidase-like enzyme in endothelial cell plasma membrane. Am J Respir Cell Mol Biol 12: 41-49, 1995. 
33. Bernard GR, Wheeler AP, Arons MM, et al: A trial of antioxidants $\mathrm{N}$-acetylcysteine and procysteine in ARDS. The Antioxidant in ARDS Study Group. Chest 112: 164-172, 1997.

34. Takao Y, Mikawa K, Nishina K and Obara H: Attenuation of acute lung injury with propofol in endotoxemia. Anesth Analg 100: 810-816, 2005.

35. Shao H, Li J, Zhou Y, et al: Dose-dependent protective effect of propofol against mitochondrial dysfunction in ischaemic/reperfused rat heart: role of cardiolipin. Br J Pharmacol 153: 1641-1649, 2008.

36. Wang B, Shravah J, Luo H, Raedschelders K, Chen DD and Ansley DM: Propofol protects against hydrogen peroxide-induced injury in cardiac $\mathrm{H} 9 \mathrm{c} 2$ cells via Akt activation and $\mathrm{Bcl}-2$ up-regulation. Biochem Biophys Res Commun 389: 105-111, 2009.
37. Ware LB: Pathophysiology of acute lung injury and the acute respiratory distress syndrome. Semin Respir Crit Care Med 27: 337-349, 2006.

38. Park HR, Kamau PW and Loch-Caruso R: Involvement of reactive oxygen species in brominated diphenyl ether-47-induced inflammatory cytokine release from human extravillous trophoblasts in vitro. Toxicol Appl Pharmacol 274: 283-292, 2014.

39. Luo C, Yuan D, Li X, et al.: Propofol Attenuated Acute Kidney Injury after Orthotopic Liver Transplantation via Inhibiting Gap Junction Composed of Connexin 32. Anesthesiology: Sept 24 2014 (Epub ahead of print).

40. Ma L, Wu X, Chen W and Fujino Y: Propofol has anti-inflammatory effects on alveolar type II epithelial cells. Acta anaesthesiologica Scandinavica 54: 362-369, 2010. 